\title{
Time-Domain Interleaved High Sampling Rate System for Large Signal Characterization of Non-Linear Devices
}

\author{
S. Ahmed, G. Neveux, T. Reveyrand, D. Barataud, and J. M. Nebus \\ XLIM, University of Limoges, France.
}

\begin{abstract}
This paper proposes a high sampling rate, 4channel Track and Hold Amplifier based time-domain measurement setup. It achieves an equivalent high sampling rate using Coherent Time Interleaved Sampling (CTIS) technique to accurately characterize high Power amplifiers driven by repetitive pulsed RF large signal. The acquired samples are the combination of non-sequential sub-sampling technique and the measurement system based on the use of Track and Hold amplifiers to avoid Intermediate Frequency (IF) aliasing and IF filtering. This principle is applied to the large signal pulsed characterization of S-band 50 W GaN amplifier. Fully calibrated acquired time-domain waveforms and power characteristics under varying load conditions are presented. To the authors knowledge the transients at the beginning and end of pulses are measured and exhibited for the first time.
\end{abstract}

Index Terms - Time-domain measurements, Track and hold amplifier, virtual sampling Frequency, Non-Linear devices.

\section{INTRODUCTION}

Incessant demands for high power and ultra wideband communication systems have encouraged characterizing nonlinear wide band gap devices and system under large signals. These high power devices depict undesired effects when pushed to saturation. Among non-linear devices transient phenomenon is of prime importance when talking about short term memory effects. To observe these transient characteristics time-domain measurement approach is the only solution to retrieve sufficient information

Many time-domain measurement systems based on different techniques have been developed in the past. All these systems translate the fundamental radio spectra into intermediate frequency spectra using sampler based [1], mixer based or most recently Track and Hold Amplifier (THA) [2], [3] based approach. THA based time-domain measurement system applies direct voltage sampling technique [2] compared to charge sampling technique [2] used in samplers. The use of continuous sinusoidal clock in contrast to pulsed strobe signal in samplers, reduce the circuit complexities and completely eradicate the IF circuitry.

For the characterization of non-linear devices producing complex harmonics spectrum, the sub-sampling systems are bandwidth limited by the maximum sampling rate of high dynamic range analog to digital convertors (ADC). However to visualize transients at RF very high sampling frequencies far beyond the Nyquist criterion should be used. Using time interleaving method in a repetitive signal is a well known technique to achieve an equivalent high sampling rate in high dynamic range ADC's. This method is easy to implement in the proposed THA based time-domain measurement system because it utilizes a common clock for direct RF sampling and digitizing.

The first section of the paper describes the time interleaved sampling method followed by the measurement system description in section two. Pulsed time-domain measurement results applied to 50W GaN PA for different load are presented in the last part.

\section{COHERENT TIME INTERLEAVED SAMPLING}

A- Introduction to Sub-sampling Principle

This work has been done with intent to increase the overall direct sampling rate to measure the RF signal by satisfying the Nyquist criterion using the following equation.

$$
\begin{aligned}
& \mathrm{F}_{\mathrm{s}-\mathrm{RF}}=\mathrm{n}^{*} \mathrm{~F}_{\mathrm{RF}} \quad \mathrm{n}>>2 \\
& \text { Where } \\
& \mathrm{F}_{\mathrm{RF}}=\text { fundamental RF frequency } \\
& \mathrm{F}_{\mathrm{s}-\mathrm{RF}}=\text { Direct RF Sampling frequency } \\
& \mathrm{n}=\text { Number of desirable samples per RF period }
\end{aligned}
$$

Practical implementation of this equation is prohibitive at RF frequencies due to front end bandwidth limitations. Subsampling method is one of the solutions to implement an equivalent high sampling rate for periodic signals respecting eq. 1 as followed:

$$
\mathrm{F}_{\mathrm{e}-\mathrm{RF}}=\mathrm{n} * \mathrm{~F}_{\mathrm{RF}}
$$

Where

$\mathrm{F}_{\mathrm{e}-\mathrm{RF}}=$ Equivalent RF Sampling frequency

To obtain this equivalent RF sampling rate, measurement systems use sub-sampling principle based on real sampling frequency defined by the following equations

$$
\begin{aligned}
& \mathrm{T}_{\mathrm{RS}}=\mathrm{T}_{\mathrm{FRAME}}+\mathrm{T}_{\mathrm{e}-\mathrm{RF}} \\
& \mathrm{T}_{\mathrm{FRAME}}=\mathrm{N}_{\mathrm{TRF}} * \mathrm{~T}_{\mathrm{RF}} \quad\left(\mathrm{T}_{\mathrm{FRAME}}>\mathrm{T}_{\mathrm{RF}}\right)
\end{aligned}
$$

Where

$\mathrm{T}_{\mathrm{RS}}=$ time period of real sampling frequency

$\mathrm{N}_{\mathrm{TRF}}=$ an integer equal to the number of fundamental $\mathrm{RF}$ periods falling in the frame period $\mathrm{T}_{\mathrm{FRAME}}$.

The implementation of these equations corresponds to a stroboscopic sample acquisition where samples are sequentially acquired. The main drawback of this sequential method is that the real sampling frequency $\left(\mathrm{F}_{\mathrm{RS}}\right)$ could be very low. Consequently, acquisition time required to visualize an image of the desired RF signal is very large when the $T_{e-R F}$ tends to 0 .

To find a trade-off between the acquisition time and the real sampling frequency, Coherent Time Interleaved Sampling (CTIS) is developed [5]. The main idea is to divide $\mathrm{T}_{\text {FRAME }}$ 
into number of segments $\mathrm{K}$ which is also an integer (maintaining the coherence of the sampling acquisition process).

$\mathrm{T}_{\text {SEGMENT }}=\mathrm{T}_{\mathrm{FRAME}} / \mathrm{K}$

So, eq. 3 can be re-defined by changing $T_{\text {FRAME }}$ to $T_{\text {SEGMENT }}$ as:

$$
\mathrm{T}_{\mathrm{RS}}=\mathrm{T}_{\mathrm{SEGMENT}}+\mathrm{T}_{\mathrm{e}-\mathrm{RF}}
$$

The repercussion of this change is that the acquired samples are scrambled in time and require re-ordering but the acquisition time is reduced by a factor of $\mathrm{K}$. Time interleaved samples acquisition was performed over number of frames and finally all the scrambled samples within all frames are reordered to represent one frame as described in Fig 1.

The number of required samples $\left(\mathrm{N}_{\text {SAMPLES }}\right)$ necessary to perform interleaving is equal to:

$$
\mathrm{N}_{\text {SAMPLES }}=\mathrm{n}^{*} \mathrm{~N}_{\mathrm{TRF}}
$$

Introducing eq. 4, 5 and 7 in eq. 6 leads to the representative equation of the coherent time interleaving process:

$$
\mathrm{T}_{\mathrm{RS}}=\mathrm{T}_{\mathrm{V}-\mathrm{RF}} *\left[\left(\mathrm{~N}_{\mathrm{SAMPLES}} / \mathrm{K}\right)+1\right]
$$

This real sampling time is used to acquire the desired number of samples of the signal under test and represent a frame period as set initially. Number of frames $\left(\mathrm{N}_{\mathrm{FR}}\right)$ falling into the acquisition interval before interleaving could be determined by the following eq. 9

$$
\mathrm{T}_{\mathrm{RS}} / \mathrm{T}_{\mathrm{FRAME}}=\mathrm{N}_{\mathrm{FR}} / \mathrm{N}_{\mathrm{SAMPLES}}
$$

The samples acquired are not in the proper order so reordering of the samples is required once the acquisition is complete. The first step to re-order samples is to determine the beginning and end time of each frame and calculate the shift of first sample (eq. 10) corresponding to each frame from its beginning time instant.

$[\text { Shift }]_{\mathrm{nFR}}=\left[\mathrm{t}_{\mathrm{s} \mathrm{nFR}}-\mathrm{T}_{\mathrm{FRAME}}\left(\mathrm{i}_{\mathrm{FR}}-1\right)_{\mathrm{nFR}} / \mathrm{T}_{\mathrm{e}-\mathrm{RF}}\right]$

Where

$$
\begin{aligned}
& t_{S n F R}=\text { time of first sample corresponding to } \\
& \text { frame }{ }^{\circ} n_{F R} \\
& i_{F R}=\text { index of frame } \\
& n_{F R} \text { varies from } 1 \text { to } N_{F R}
\end{aligned}
$$

As due to the non coherent sampling frequency the number of samples in each frame is not constant and is determined by the following equation 11 .

$[\mathrm{Nbr}]_{\mathrm{nFR}}=\left[\left(\mathrm{i}_{\mathrm{EFR}}-\mathrm{i}_{\mathrm{BFR}}\right)_{\mathrm{nFR}}+1\right]$

Where

$\mathrm{Nbr}=\mathrm{an}$ array of number of samples in each frame $\mathrm{i}_{\mathrm{EFR}}=$ index of sample at end of each frame $\mathrm{i}_{\mathrm{BFR}}=$ index of sample at beginning of each frame

The last step is to re-order (eq. 12) the sample points using the calculated information:

$\left[\mathrm{New} \_\right.$index $]=\left[1: \mathrm{N}_{\mathrm{FR}}:\left(\mathrm{N}_{\mathrm{NFR}} * \mathrm{Nbr}_{\mathrm{nFR}}\right)\right]+[\text { Shift }]_{\mathrm{nFR}}$
The Fig. 1 explains an example of CTIS system for a fundamental $\mathrm{RF}\left(\mathrm{F}_{\mathrm{O}}\right)$ of $125 \mathrm{MHz}$ and the sampling rate to be $8 \mathrm{~F}_{\mathrm{O}}$. Frame period was chosen to be $8 \mathrm{~ns}$ which ensures only one RF period within the frame. Segment time was chosen as 4 ns which leads that there are two segments within the frame. Total number of samples to be acquired to represent one frame was equal to 8 . After the CTIS calculation real sampling frequency (eq. 8) was found to be $5 \mathrm{~ns}$.

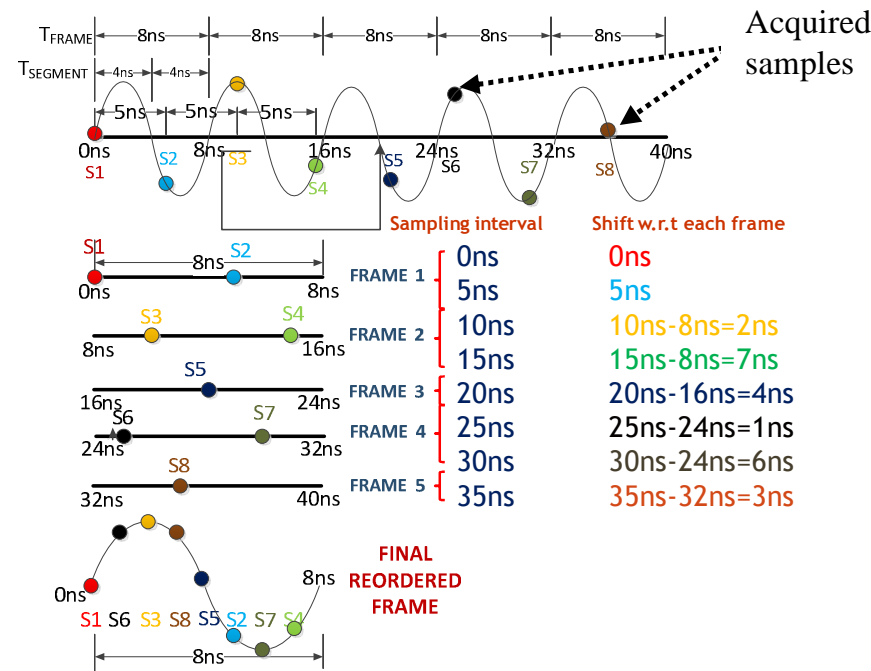

Fig. 1. An example representing coherent interleaved sampling phenomenon

\section{MEASUREMENT SYSTEM DESCRIPTION}

The 4-channel time-domain measurement system employing time-interleaving principle is described in Fig. 2.

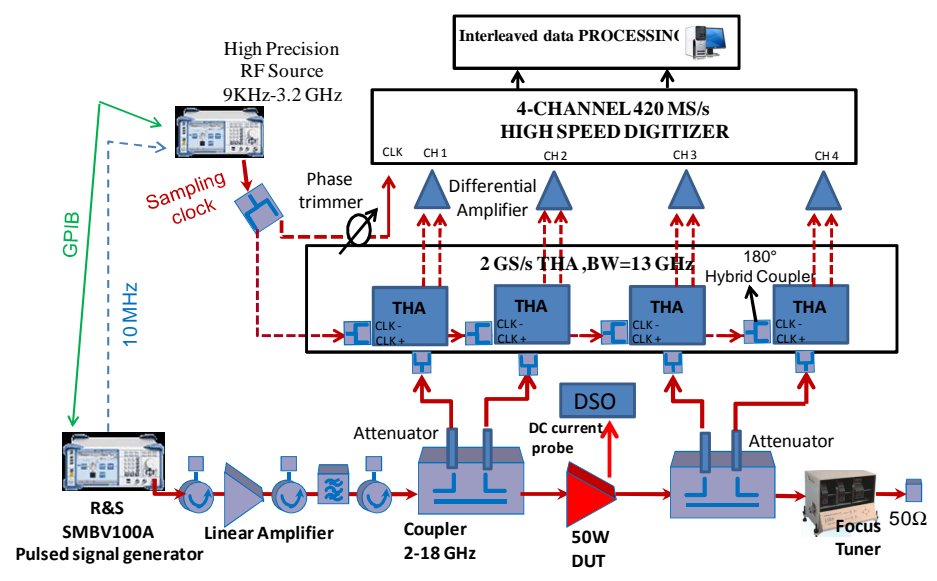

Fig. 2. 4-channel time-domain measurement system description

A signal modulated unit is used to generate $\mathrm{RF} \mathrm{CW}$ or Modulated signals. The signal is linearly amplified and feeds the input of the power amplifier under test (AUT). The incident and reflected signals at the input and output of DUT are measured at the same instant by using two directional couplers, and four $13 \mathrm{GHz}$ bandwidth 2 GS/s THAs. THAs are 
followed by differential amplifiers and a four channel $420 \mathrm{MS} / \mathrm{s}$, high dynamic range (12 bit) ADC. A common high precision sinusoidal clock configured by the previously described CTIS calculation is fed into the THA and the ADC. The RF signals are then directly sampled by the THA and the ADC synchronized by a $10 \mathrm{MHz}$ signal. The acquired data is then processed to reorder the samples as described in section II. This is where this measurement system is different to the one described in [2], [3], [4]. In [2] sub-sampling technique was used to down convert the RF signal and then sampled at four times the IF frequency. The in phase and quadrature components were computed and the phase and amplitude envelope (Fig. 3) information was demodulated from the pulsed signal.

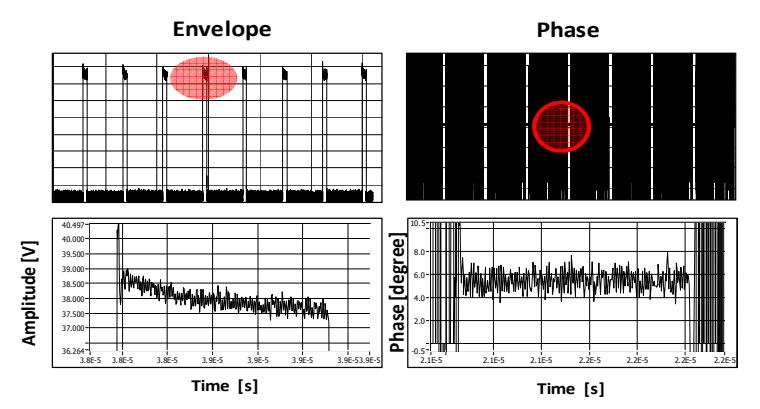

Fig. 3. Measured time-domain pulsed output waveform and extracted pulsed envelop (without DC component) and phase information [2]

\section{A. System Calibration Procedure}

An absolute SOLT system calibration was performed to mathematically remove systematic errors. The goal of the calibration procedure was to determine the eight unknown complex error coefficients for each frequency (fundamental and harmonics) [6].

$$
\left[\begin{array}{l}
\boldsymbol{a}_{1}^{i} \\
\boldsymbol{b}_{1}^{i} \\
\boldsymbol{a}_{2}^{i} \\
\boldsymbol{b}_{2}^{i}
\end{array}\right]_{D S T}=\boldsymbol{K}^{i} \exp \left(\boldsymbol{j} \boldsymbol{\varphi}^{j}\right)\left[\begin{array}{cccc}
1 & \boldsymbol{\beta}_{1}^{i} & 0 & 0 \\
\boldsymbol{\gamma}_{1}^{j} & \boldsymbol{\delta}_{1}^{i} & 0 & 0 \\
0 & 0 & \boldsymbol{\alpha}_{2}^{i} & \boldsymbol{\beta}_{2}^{i} \\
0 & 0 & \boldsymbol{\gamma}_{2}^{i} & \boldsymbol{\delta}_{2}^{i}
\end{array}\right]\left[\begin{array}{l}
\boldsymbol{a}_{1 M}^{i} \\
\boldsymbol{b}_{1 M}^{i} \\
\boldsymbol{a}_{2 M}^{i} \\
\boldsymbol{b}_{2 M}^{i}
\end{array}\right]
$$

The first three steps are similar to the calibration procedure performed for classical vector network analyzer by connecting priori known short, open and load coefficients to port 1 and port 2 respectively. Next a through connection is made between the two ports. These error coefficients associate raw data provided by ADC measurement channels to the incident and scattered power waves at the input and output of device under test. Next step is to determine the K coefficient which is also a complex number. Its amplitude is found by connecting a power meter to the port one and measurement is made for each frequency. Then a previously calibrated [357-536 MHz] comb generator has been used as harmonic phase reference to correct the phase of the proposed measurement system. For modulated signals the assumption of a constant group delay of the 4 channels of the receiver was made over the envelop bandwidth.

\section{Pulsed MEASUREMENT RESUlts}

The proposed CTIS based test bench has been applied to the characterization of 50W GaN Nitronex (NPTB00050B) PA under varying loads. It was to testify the capabilities of measurement system in load-pull environment and to observe the RF time domain voltage and current waveforms at both ports of the amplifiers. The PA was operating at a drain quiescent current of $330 \mathrm{~mA}$ and a drain voltage of $28 \mathrm{~V}$. A pulse modulated signal with period of 50 us and $20 \%$ duty cycle was applied to the PA at a fundamental frequency of $2.5 \mathrm{GHz}$. The sampling rate was chosen to be 10 times the fundamental frequency. Frame period $\left(\mathrm{T}_{\mathrm{FRAME}}\right.$ ) of $50 \mathrm{us}$ second was chosen which means that $62500 \mathrm{RF}$ periods could be acquired in the pulse width as represented in Fig. 4. $\mathrm{T}_{\text {SEGMENT }}$ was chosen to be $20 \mathrm{~ns}$ to adapt the real sampling frequency near $50 \mathrm{MHz}$. Total samples were captured in 501 frames and the samples were reordered to represent one frame of RF signal with 1.25 million samples at a high sampling frequency of $25 \mathrm{GHz}$ (10 samples per RF period).
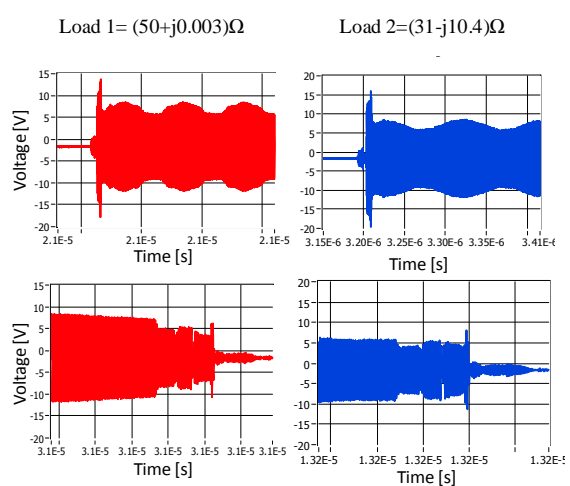

Load $2=(63.7-\mathrm{j} 23.9) \Omega$

Fig. 4. Input voltage transients at the beginning and end of pulse under varying loads

Fig. 4 represents the input voltage transient at the beginning and end of the pulse and during the three measurements performed at varying loads. Almost no change in the transients for the input voltages is noticed.

Fig. 5 represents the evolution of the directly sampled RF voltages and current waveforms for variable loads and a fixed power level (1 dB compression). Pulsed output voltages and current waveforms of the amplifier under test (AUT) represent that no harmonics are present even at $1 \mathrm{~dB}$ compression point. (presence of band-pass filter matching circuits at the output) 


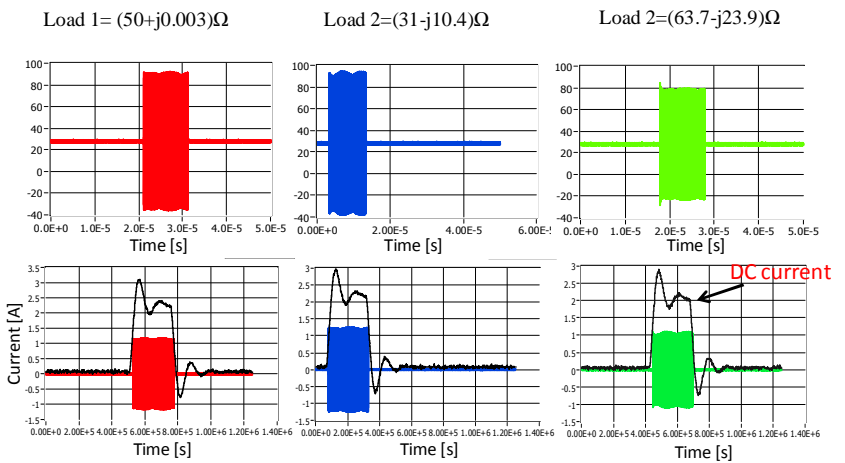

Fig. 5. RF and DC measured pulsed output voltage and current of AUT at different loads.

The DC current during the pulse is also measured using a digital storage oscilloscope. The fact that the RF receiver based on THA and DSO does not have same number of samples making it difficult to add the DC and RF current. They are superposed on Fig. 5.

Fig. 6 represent the RF output voltage transients at beginning and end of the pulse under varying loads and a clear difference could be noticed in the amplitude and the transient behavior. This Figure confirms that the AUT and the memory effects occurring in this nonlinear device are very sensitive to the loads.

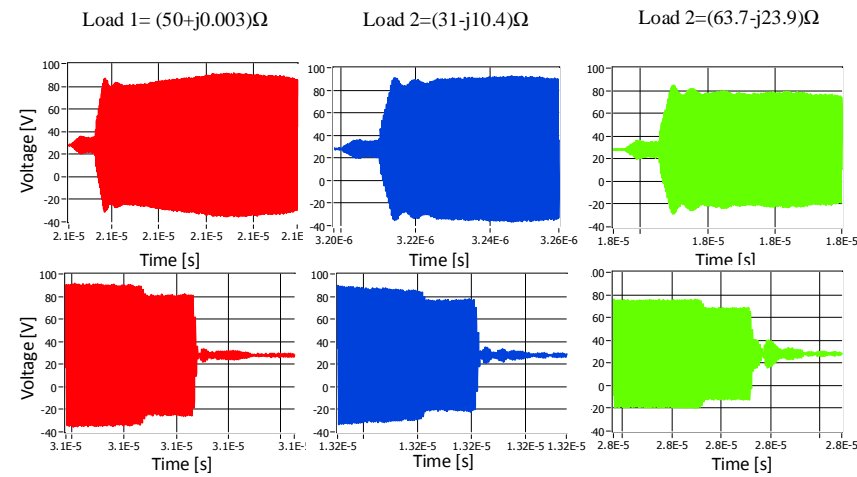

Fig. 6. Output voltage transients at the beginning and end of pulse under varying loads

\section{Conclusion}

This work represents a fully calibrated THA based 4channel time-domain high sampling rate pulsed measurement test bench. High sampling rate $(25 \mathrm{GHz}$ equivalent frequency sampling) is achieved by time interleaved acquisition of samples. A process has been developed to reorder the scrambled samples to represent the directly sampled RF signal. Time-domain characterization of high power $\mathrm{GaN}$ under varying loads has been performed. Considerable differences in RF power characteristics, voltage and current transients are clearly observed. To the authors knowledge this
$\mathrm{RF}$ transient phenomenon is measured and represented for the first time.

\section{REFERENCES}

[1] K. Andersson, M. Thorsell,G. Pailloncy and F. Verbeyst "Largesignal waveform acquisition of pulsed signals,"41st European Microwave conference, Manchester, 10-13 ${ }^{\text {th }}$ October 2011.

[2] S. Ahmed, M. Saad-el-dine, G. Neveux, D. Barataud, J. Nebus"4-Channel, High Dynamic Range Time-Domain Measurement System Using Track \& Hold Amplifier Utilized for the Characterization and Linearization of High Power GaN Amplifiers" International Journal of Microwave and Wireless Technologies, volume 4, issue 01, pp. 71-79.

[3] S. Ahmed, M. Saad-el-Dine, G. Neveux, T. Reveyrand, D. Barataud, J. Nebus "Time-Domain Measurement System Using Track \& Hold Amplifier Applied to Pulsed RF Characterization of High Power GaN Devices" IEEE International Microwave symposium Baltimore 2011.

[4] J. Faraj, Guillaume Callet, Fabien De Groote, Jan Verspecht, Raymond Quere, Jean Pierre Teyssier "Burst of Pulses For Time domain Large Signal Measurments", ARFTG International Microwave Symposium Boston USA, 2009.

[5] Lecroy corp. "Coherent Interleaving Sampling," patent: WO/2006/086257, August, 2006.

[6] Verspecht "Calibration of a measurement system for high frequency non-linear devices", Doctoral Dissertation - Vrije universiteit Brussel, November 1995. 